\title{
Timoshenko systems with fading memory
}

\author{
Monica Conti, Filippo Dell'Oro, and Vittorino Pata
}

Communicated by Sergey Zelik, received September 18, 2013.

\begin{abstract}
The decay properties of the semigroup generated by a linear Timoshenko system with fading memory are discussed. Uniform stability is shown to occur within a necessary and sufficient condition on the memory kernel $\mu$.
\end{abstract}

\section{CONTEnTs}

1. Introduction 367

2. Functional Setting and Notation 368

3. The Contraction Semigroup $\quad 369$

4. Theorem 1 (Necessity) 370

5. Theorem 1 (Sufficiency) 371

$\begin{array}{ll}\text { References } & 376\end{array}$

\section{Introduction}

Given a real interval $\mathfrak{I}=[0, \ell]$, we consider the viscoelastic beam model of Timoshenko type $[\mathbf{1 2}]$

$$
\left\{\begin{array}{l}
\rho_{1} \varphi_{t t}-\kappa\left(\varphi_{x}+\psi\right)_{x}=0 \\
\rho_{2} \psi_{t t}-b \psi_{x x}+\int_{0}^{\infty} \mu(s) \psi_{x x}(t-s) \mathrm{d} s+\kappa\left(\varphi_{x}+\psi\right)=0
\end{array}\right.
$$

in the unknowns $\varphi, \psi:(x, t) \in \mathfrak{I} \times[0, \infty) \mapsto \mathbb{R}$, where the strictly positive constants $\rho_{1}, \rho_{2}, \kappa, b$ satisfy the relation

$$
\frac{\rho_{1}}{\kappa}=\frac{\rho_{2}}{b}
$$

Key words and phrases. Timoshenko system, fading memory, contraction semigroup, exponential stability. 
while the memory kernel $\mu$ is a (nonnegative) nonincreasing absolutely continuous function on $[0, \infty)$ such that

$$
a:=b-m>0 \quad \text { where } \quad m:=\int_{0}^{\infty} \mu(s) \mathrm{d} s>0 .
$$

The system is complemented with the Dirichlet boundary conditions

$$
\varphi(0, t)=\varphi(\ell, t)=\psi(0, t)=\psi(\ell, t)=0,
$$

but our arguments can be used to prove analogous results for other kind of boundary conditions as well, such as Dirichlet/Neumann or Neumann/Dirichlet.

Following [7], by rephrasing the problem within the history framework of Dafermos [3], system (1.1) is shown to generate a contraction semigroup $S(t)$ of solutions acting on a suitable Hilbert space $\mathcal{H}$ accounting for the presence of the memory. The aim of this work is to establish a necessary and sufficient condition on the memory kernel $\mu$ (within the class of kernels considered above) in order for $S(t)$ to be exponentially stable on $\mathcal{H}$, namely,

$$
\|S(t) z\|_{\mathcal{H}} \leq K \mathrm{e}^{-\omega t}\|z\|_{\mathcal{H}}, \quad \forall z \in \mathcal{H}
$$

for some $K \geq 1$ and $\omega>0$. Our main theorem reads as follows.

TheOREM 1. The semigroup $S(t)$ is exponentially stable if and only if there exist $C \geq 1$ and $\delta>0$ such that

$$
\mu(\sigma+s) \leq C \mathrm{e}^{-\delta \sigma} \mu(s)
$$

for every $\sigma \geq 0$ and $s>0$.

The decay properties of $S(t)$ have been previously studied in the papers $[\mathbf{6}, \mathbf{7}]$. We will discuss and compare those results in the next Sections 4 and 5 , where we will also provide the proofs of the two directions of Theorem 1. Condition (1.3) appears for the first time in connection with systems with memory in $[\mathbf{2}]$, whereas (1.2), which basically says that the two hyperbolic equations share the same propagation speed, is used in [1] for the same model but with a convolution integral of Volterra type. In that work, the failure of (1.2) is shown to prevent the possibility of any uniform decay of the solutions. Actually, the same phenomenon pops up if the convolution integral, which contains the whole dissipation of the system, is replaced by an instantaneous damping term, such as $\psi_{t}$ (see [11]).

REMARK. The existence of the semigroup $S(t)$ can be actually established under weaker conditions on $\mu$, which can be only piecewise absolutely continuous with (infinitely many) discontinuity points. For the proof of the necessity part of Theorem 1 nothing more is needed. In particular, no use is made of (1.2). Concerning sufficiency, besides (1.2)-(1.3), one has to require that the set where $\mu^{\prime}<0$ has positive measure, automatically satisfied if $\mu$ is absolutely continuous (as in our hypotheses).

\section{Functional Setting and Notation}

In what follows, $\langle\cdot, \cdot\rangle$ and $\|\cdot\|$ are the inner product and norm on the (real) Hilbert space $L^{2}(\mathfrak{I})$. We will also consider the Sobolev space $H_{0}^{1}(\mathfrak{I})$ endowed with the 
gradient norm, due to the Poincaré inequality, along with the $L^{2}$-weighted space of $H_{0}^{1}$-valued functions on $\mathbb{R}^{+}=(0, \infty)$

$$
\mathcal{M}=L^{2}\left(\mathbb{R}^{+} ; H_{0}^{1}(\mathfrak{I})\right), \quad\langle\eta, \xi\rangle_{\mathcal{M}}=\int_{0}^{\infty} \mu(s)\left\langle\eta_{x}(s), \xi_{x}(s)\right\rangle \mathrm{d} s .
$$

We define the linear operator $T$ on $\mathcal{M}$ by

$$
T \eta=-D \eta, \quad \mathfrak{D}(T)=\{\eta \in \mathcal{M}: D \eta \in \mathcal{M}, \eta(0)=0\},
$$

where $D$ stands for weak derivative. The operator $T$ is the infinitesimal generator of the right-translation semigroup $\Sigma(t)$ on $\mathcal{M}$, acting as

$$
[\Sigma(t) \eta](s)= \begin{cases}0 & s \leq t, \\ \eta(s-t) & s>t .\end{cases}
$$

The phase space of our problem will be ${ }^{1}$

$$
\mathcal{H}=H_{0}^{1}(\mathfrak{I}) \times L^{2}(\mathfrak{I}) \times H_{0}^{1}(\mathfrak{I}) \times L^{2}(\mathfrak{I}) \times \mathcal{M}
$$

normed by

$$
\|(\varphi, \tilde{\varphi}, \psi, \tilde{\psi}, \eta)\|_{\mathcal{H}}^{2}=\kappa\left\|\varphi_{x}+\psi\right\|^{2}+\rho_{1}\|\tilde{\varphi}\|^{2}+a\left\|\psi_{x}\right\|^{2}+\rho_{2}\|\tilde{\psi}\|^{2}+\|\eta\|_{\mathcal{M}}^{2} .
$$

Finally, we recall [2, Theorem 3.3].

TheOREM 2. The right-translation semigroup $\Sigma(t)$ acting on $\mathcal{M}$ is exponentially stable if and only if (1.3) holds.

\section{The Contraction Semigroup}

We formally define the auxiliary variable $\eta=\eta^{t}(s)$ as (the dependence on $x$ is omitted)

$$
\eta^{t}(s)=\psi(t)-\psi(t-s)
$$

Then, (1.1) turns into the system in the unknowns $\varphi=\varphi(t), \psi=\psi(t)$ and $\eta=\eta^{t}$

$$
\left\{\begin{array}{l}
\rho_{1} \varphi_{t t}-\kappa\left(\varphi_{x}+\psi\right)_{x}=0 \\
\rho_{2} \psi_{t t}-a \psi_{x x}-\int_{0}^{\infty} \mu(s) \eta_{x x}(s) \mathrm{d} s+\kappa\left(\varphi_{x}+\psi\right)=0 \\
\eta_{t}=T \eta+\psi_{t} .
\end{array}\right.
$$

Introducing the state vector

$$
Z(t)=\left(\varphi(t), \tilde{\varphi}(t), \psi(t), \tilde{\psi}(t), \eta^{t}\right)
$$

system (3.1) can be clearly written as a linear ODE in $\mathcal{H}$ of the form

$$
\frac{\mathrm{d}}{\mathrm{d} t} Z(t)=\mathbb{A} Z(t)
$$

where the domain $\mathfrak{D}(\mathbb{A})$ of the linear operator $\mathbb{A}$, whose action can be easily deduced from $(3.1)$, is made by all the vectors $(\varphi, \tilde{\varphi}, \psi, \tilde{\psi}, \eta) \in \mathcal{H}$ such that

$$
\varphi, \psi \in H^{2}(\mathfrak{I}), \quad \tilde{\varphi}, \tilde{\psi} \in H_{0}^{1}(\mathfrak{I}), \quad \eta \in \mathfrak{D}(T), \quad \int_{0}^{\infty} \mu(s) \eta(s) \mathrm{d} s \in H^{2}(\mathfrak{I}) .
$$

\footnotetext{
${ }^{1}$ In the case of Neumann boundary conditions, one has to work in spaces of zero-mean functions.
} 
According to $[\mathbf{7}]$, the operator $\mathbb{A}$ is the infinitesimal generator of a contraction semigroup

$$
S(t)=\mathrm{e}^{t \mathbb{A}}: \mathcal{H} \rightarrow \mathcal{H}
$$

In particular, $\mathbb{A}$ is dissipative. Indeed, for every $z=(\varphi, \tilde{\varphi}, \psi, \tilde{\psi}, \eta) \in \mathfrak{D}(A)$,

$$
\langle\mathbb{A} z, z\rangle_{\mathcal{H}}=\langle T \eta, \eta\rangle_{\mathcal{M}}=\frac{1}{2} \int_{0}^{\infty} \mu^{\prime}(s)\left\|\eta_{x}(s)\right\|^{2} \mathrm{~d} s \leq 0 .
$$

Thus, for every initial datum $z=\left(\varphi_{0}, \tilde{\varphi}_{0}, \psi_{0}, \tilde{\psi}_{0}, \eta_{0}\right) \in \mathcal{H}$ given at time $t=0$, the unique solution at time $t>0$ to $(3.2)$ reads

$$
Z(t)=\left(\varphi(t), \varphi_{t}(t), \psi(t), \psi_{t}(t), \eta^{t}\right)=S(t) z,
$$

where $\eta^{t}$ fulfills the explicit representation formula (see [5])

$$
\eta^{t}(s)= \begin{cases}\psi(t)-\psi(t-s) & s \leq t, \\ \eta_{0}(s-t)+\psi(t)-\psi_{0} & s>t .\end{cases}
$$

\section{Theorem 1 (Necessity)}

The proof of the necessity part of Theorem 1 is essentially the same of $[\mathbf{2}$, Theorem 3.2], dealing with a linearly viscoelastic equation. For the reader's convenience, we report here the short argument.

Proof of Theorem 1 (Necessity). Suppose $S(t)$ exponentially stable on $\mathcal{H}$. Then, for any initial datum $z \in \mathcal{H}$ of the form $z=\left(0,0,0,0, \eta_{0}\right)$ we have

$$
\max \left\{\left\|\psi_{x}(t)\right\|,\left\|\eta^{t}\right\|_{\mathcal{M}}\right\} \leq \max \{1 / a, 1\}\|S(t) z\|_{\mathcal{H}} \leq K \mathrm{e}^{-\omega t}\left\|\eta_{0}\right\|_{\mathcal{M}}
$$

for some positive $K, \omega$. On the other hand, exploiting the representation formula (3.4),

$$
\begin{aligned}
2\left\|\eta^{t}\right\|_{\mathcal{M}}^{2} & \geq 2 \int_{t}^{\infty} \mu(s)\left\|\eta_{0 x}(s-t)+\psi_{x}(t)\right\|^{2} \mathrm{~d} s \\
& \geq \int_{t}^{\infty} \mu(s)\left\|\eta_{0 x}(s-t)\right\|^{2} \mathrm{~d} s-2 m\left\|\psi_{x}(t)\right\|^{2} \\
& \geq\left\|\Sigma(t) \eta_{0}\right\|_{\mathcal{M}}^{2}-2 m K^{2} \mathrm{e}^{-2 \omega t}\left\|\eta_{0}\right\|_{\mathcal{M}}^{2} .
\end{aligned}
$$

We conclude that

$$
\left\|\Sigma(t) \eta_{0}\right\|_{\mathcal{M}} \leq K \sqrt{2(1+m)} \mathrm{e}^{-\omega t}\left\|\eta_{0}\right\|_{\mathcal{M}}
$$

and the claim is a consequence of Theorem 2 .

Our Theorem 1 (Necessity), seems to contradict the following result established by Messaoudi and Said-Houari:

Theorem 3 (Theorem 2.1 in [6]). Let $\mu$ satisfy the differential inequality

$$
\mu^{\prime}(s)+\delta[\mu(s)]^{p} \leq 0
$$

for some $\delta>0$ and some $p \in\left(1, \frac{3}{2}\right)$. Then, for every initial datum $z \in \mathcal{H}$, the inequality

$$
E(t):=\frac{1}{2}\|S(t) z\|_{\mathcal{H}}^{2} \leq \frac{M}{(1+t)^{1 /(p-1)}}
$$

holds for some $M>0$ depending on $z$. 
Let us observe that the correct conclusion of Theorem 3 should have been that the energy decays exponentially, for lack of exponential stability prevents the existence of uniform decay patterns. Indeed, the thesis of Theorem 3 implies that, for every $z \in \mathcal{H}$,

$$
\left\|(1+t)^{1 /(2 p-2)} S(t) z\right\|_{\mathcal{H}} \leq Q_{z}
$$

for some $Q_{z}>0$, and a direct application of the Uniform Boundedness Principle yields

$$
\|S(t) z\|_{\mathcal{H}} \leq \frac{Q}{(1+t)^{1 /(2 p-2)}}\|z\|_{\mathcal{H}},
$$

where $Q>0$ is now independent of $z$. Hence the operator norm of $S(t)$ goes below one for large values of $t$, and exponential stability readily follows. At the same time, $S(t)$ cannot have a uniform decay if, for instance,

$$
\mu(s)=\frac{1}{(1+s)^{1 /(p-1)}},
$$

which complies with (4.1) but clearly violates (1.3).

\section{Theorem 1 (Sufficiency)}

The exponential stability of $S(t)$ has been proved in [7] within the hypotheses

$$
\mu^{\prime}(s)+k_{1} \mu(s) \geq 0, \quad\left|\mu^{\prime \prime}(s)\right| \leq k_{2} \mu(s),
$$

for some $k_{1}, k_{2}>0$, and

$$
\mu^{\prime}(s)+\delta \mu(s) \leq 0,
$$

for some $\delta>0$. Let aside (5.1), which is only technical, condition (5.2) is equivalent to (1.3) with $C=1$. Nonetheless, (1.3) with $C>1$ turns out to be much more general than (5.2). For instance, any compactly supported $\mu$ (in the class of kernels considered in the present paper) satisfies (1.3), but cannot comply with (5.2) if it has flat zones, or even horizontal inflection points. Besides, (1.3) with $C>1$ makes no assumptions at all on the derivative $\mu^{\prime}$.

Analogously to [7], the proof of the sufficiency part of Theorem 1 is based on the following abstract result from [10] (see also [4] for the precise statement used here).

LEMma 4. The contraction semigroup $S(t)$ on $\mathcal{H}$ is exponentially stable if and only if there exists $\varepsilon>0$ such that

$$
\inf _{\lambda \in \mathbb{R}}\|\mathrm{i} \lambda z-\mathbb{A} z\|_{\mathcal{H}} \geq \varepsilon\|z\|_{\mathcal{H}}, \quad \forall z \in \mathfrak{D}(\mathbb{A}),
$$

where $\mathbb{A}$ and $\mathcal{H}$ are understood to be the complexifications of the original $\mathbb{A}$ and $\mathcal{H}$.

Proof of Theorem 1 (Sufficiency). Within hypothesis (1.3), suppose $S(t)$ be not exponentially stable. Then, Lemma 4 ensures the existence of sequences $\lambda_{n} \in \mathbb{R}$ and $z_{n}=\left(\varphi_{n}, \tilde{\varphi}_{n}, \psi_{n}, \tilde{\psi}_{n}, \eta_{n}\right) \in \mathfrak{D}(\mathbb{A})$ with

$$
\left\|z_{n}\right\|_{\mathcal{H}}^{2}=\kappa\left\|\varphi_{n x}+\psi_{n}\right\|^{2}+\rho_{1}\left\|\tilde{\varphi}_{n}\right\|^{2}+a\left\|\psi_{n x}\right\|^{2}+\rho_{2}\left\|\tilde{\psi}_{n}\right\|^{2}+\left\|\eta_{n}\right\|_{\mathcal{M}}^{2}=1
$$

satisfying the relation

$$
\text { i } \lambda_{n} z_{n}-\mathbb{A} z_{n} \rightarrow 0 \text { in } \mathcal{H} \text {. }
$$


Componentwise, (5.3) reads

$$
\begin{aligned}
& \mathrm{i} \lambda_{n} \varphi_{n}-\tilde{\varphi}_{n} \rightarrow 0 \quad \text { in } H_{0}^{1}, \\
& \mathrm{i} \lambda_{n} \rho_{1} \tilde{\varphi}_{n}-\kappa\left(\varphi_{n x}+\psi_{n}\right)_{x} \rightarrow 0 \quad \text { in } L^{2}, \\
& \mathrm{i} \lambda_{n} \psi_{n}-\tilde{\psi}_{n} \rightarrow 0 \quad \text { in } H_{0}^{1}, \\
& \mathrm{i} \lambda_{n} \rho_{2} \tilde{\psi}_{n}-a \psi_{n x x}-\int_{0}^{\infty} \mu(s) \eta_{n x x}(s) \mathrm{d} s+\kappa\left(\varphi_{n x}+\psi_{n}\right) \rightarrow 0 \quad \text { in } L^{2}, \\
& \mathrm{i} \lambda_{n} \eta_{n}-T \eta_{n}-\tilde{\psi}_{n} \rightarrow 0 \quad \text { in } \mathcal{M} .
\end{aligned}
$$

We assume $\lambda_{n} \nrightarrow 0$ (the case $\lambda_{n} \rightarrow 0$ is much simpler and left to the reader). Accordingly, up to a subsequence (UTS in the sequel),

$$
\lambda_{n} \rightarrow \lambda_{\star} \in[-\infty, \infty] \backslash\{0\} .
$$

We will reach a contradiction by showing that every single component of $z_{n}$ goes to zero in its norm UTS. $^{2}$ The first part of the proof borrows some ideas from [9]. Since $\mu$ is an absolutely continuous function vanishing at infinity, the set

$$
\mathbb{S}=\left\{s \in \mathbb{R}^{+}: K \mu^{\prime}(s)+\mu(s)<0\right\}
$$

has positive measure for some $K>0$ large enough. Let us define the space

$$
\mathcal{S}=L_{\mu}^{2}\left(\mathbb{S} ; H_{0}^{1}(\mathfrak{I})\right)
$$

We need three preliminary lemmas. We will lean several times (without explicit mention) on the boundedness in $\mathcal{H}$ of $z_{n}$.

Lemma 5. We have the convergence $\left\|\eta_{n}\right\|_{\mathcal{S}} \rightarrow 0$.

Proof. By means of (5.3),

$$
\mathfrak{R e}\left\langle\mathrm{i} \lambda_{n} z_{n}-\mathbb{A} z_{n}, z_{n}\right\rangle_{\mathcal{H}}=-\mathfrak{R e}\left\langle\mathbb{A} z_{n}, z_{n}\right\rangle_{\mathcal{H}} \rightarrow 0,
$$

and using (3.3) we are led to

$$
0 \leq-\int_{\mathbb{S}} \mu^{\prime}(s)\left\|\eta_{n x}(s)\right\|^{2} \mathrm{~d} s \leq-\int_{0}^{\infty} \mu^{\prime}(s)\left\|\eta_{n x}(s)\right\|^{2} \mathrm{~d} s \rightarrow 0 .
$$

Since

$$
\left\|\eta_{n}\right\|_{\mathcal{S}}^{2}=\int_{\mathbb{S}} \mu(s)\left\|\eta_{n x}(s)\right\|^{2} \mathrm{~d} s \leq-K \int_{\mathbb{S}} \mu^{\prime}(s)\left\|\eta_{n x}(s)\right\|^{2} \mathrm{~d} s,
$$

the conclusion follows.

Lemma 6. The sequence $\left|\lambda_{n}\right|\left\|\tilde{\psi}_{n}\right\|_{*}$ is bounded, where $\|\cdot\|_{*}$ is the norm in $H^{-1}(\mathfrak{I})$.

Proof. By the triangle inequality,

$$
\begin{aligned}
\rho_{2}\left|\lambda_{n}\right|\left\|\tilde{\psi}_{n}\right\|_{*} \leq & \left\|\mathrm{i} \lambda_{n} \rho_{2} \tilde{\psi}_{n}-a \psi_{n x x}-\int_{0}^{\infty} \mu(s) \eta_{n x x}(s) \mathrm{d} s+\kappa\left(\varphi_{n x}+\psi_{n}\right)\right\|_{*} \\
& +\left\|a \psi_{n x x}+\int_{0}^{\infty} \mu(s) \eta_{n x x}(s) \mathrm{d} s-\kappa\left(\varphi_{n x}+\psi_{n}\right)\right\|_{*} .
\end{aligned}
$$

\footnotetext{
${ }^{2}$ It is understood that passing to a subsequence means to refine the former one.
} 
Due to (5.7) and the continuous embedding $L^{2}(\mathfrak{I}) \subset H^{-1}(\mathfrak{I})$, the first term in the right-hand side goes to zero, whereas the second one is dominated by

$$
a\left\|\psi_{n x}\right\|+\int_{0}^{\infty} \mu(s)\left\|\eta_{n x}(s)\right\| \mathrm{d} s+\kappa\left\|\varphi_{n}\right\|+\kappa\left\|\psi_{n}\right\|_{*},
$$

which is bounded uniformly with respect to $n \in \mathbb{N}$.

Lemma 7. Within (1.3), for any $\xi \in \mathcal{M}$ we have the estimate

$$
\int_{0}^{\infty} \mu(s) \int_{0}^{s}\left\|\xi_{x}(\sigma)\right\| \mathrm{d} \sigma \mathrm{d} s \leq \frac{\sqrt{4 C m}}{\delta}\|\xi\|_{\mathcal{M}} .
$$

Proof. Exploiting (1.3) and the Hölder inequality,

$$
\int_{0}^{\infty} \mu(s) \int_{0}^{s}\left\|\xi_{x}(\sigma)\right\| \mathrm{d} \sigma \mathrm{d} s \leq \sqrt{C} \int_{0}^{\infty} \sqrt{\mu(s)} G(s) \leq \sqrt{C m}\|G\|_{L^{2}\left(\mathbb{R}^{+}\right)},
$$

having set

$$
G(s)=\int_{0}^{s} \mathrm{e}^{-\frac{\delta}{2}(s-\sigma)} \sqrt{\mu(\sigma)}\left\|\xi_{x}(\sigma)\right\| \mathrm{d} \sigma \mathrm{d} s .
$$

By a well-known result of measure theory,

$$
\|G\|_{L^{2}\left(\mathbb{R}^{+}\right)} \leq \frac{2}{\delta}\|\sqrt{\mu}\| \xi_{x}\|\|_{L^{2}\left(\mathbb{R}^{+}\right)}=\frac{2}{\delta}\|\xi\|_{\mathcal{M}},
$$

which completes the argument.

We are now ready to prove the norm-decay of every single component of $z_{n}$.

LEMMA 8. The convergence $\left\|\tilde{\psi}_{n}\right\| \rightarrow 0$ holds $\mathrm{UTS}$.

Proof. Introducing $\Psi_{n}$ such that $-\Psi_{n x x}=\tilde{\psi}_{n}$, we infer from Lemmas 5 and 6 that

$$
\left|\mathrm{i} \lambda_{n}\left\langle\eta_{n}, \Psi_{n}\right\rangle_{\mathcal{S}}\right| \leq\left|\lambda_{n}\right|\left\|\tilde{\psi}_{n}\right\|_{*} \int_{\mathbb{S}} \mu(s)\left\|\eta_{n x}(s)\right\| \mathrm{d} s \leq \sqrt{m}\left|\lambda_{n}\right|\left\|\tilde{\psi}_{n}\right\|_{*}\left\|\eta_{n}\right\|_{\mathcal{S}} \rightarrow 0 .
$$

On the other hand, calling

$$
\xi_{n}=\mathrm{i} \lambda_{n} \eta_{n}-T \eta_{n}-\tilde{\psi}_{n}
$$

we find the explicit expression

$$
\eta_{n}(s)=\frac{1}{\mathrm{i} \lambda_{n}}\left(1-\mathrm{e}^{-\mathrm{i} \lambda_{n} s}\right) \tilde{\psi}_{n}+\int_{0}^{s} \mathrm{e}^{-\mathrm{i} \lambda_{n}(s-\sigma)} \xi_{n}(\sigma) \mathrm{d} \sigma .
$$

Hence,

$$
\mathrm{i} \lambda_{n}\left\langle\eta_{n}, \Psi_{n}\right\rangle_{\mathcal{S}}=\alpha_{n}\left\|\tilde{\psi}_{n}\right\|^{2}+\beta_{n} \rightarrow 0
$$

where we put

$$
\begin{aligned}
& \alpha_{n}=\int_{\mathbb{S}} \mu(s)\left(1-\mathrm{e}^{-\mathrm{i} \lambda_{n} s}\right) \mathrm{d} s, \\
& \beta_{n}=\mathrm{i} \lambda_{n} \int_{\mathbb{S}} \mu(s) \int_{0}^{s} \mathrm{e}^{-\mathrm{i} \lambda_{n}(s-\sigma)}\left\langle\xi_{n x}(\sigma), \Psi_{n x}\right\rangle \mathrm{d} \sigma \mathrm{d} s .
\end{aligned}
$$

The conclusion follows by showing that $\beta_{n} \rightarrow 0$ whereas $\alpha_{n}$ remains away from zero for large $n$. Indeed, by Lemmas 6-7 and the convergence (5.8) we get

$$
\left|\beta_{n}\right| \leq \frac{\sqrt{4 C m}}{\delta}\left|\lambda_{n}\right|\left\|\tilde{\psi}_{n}\right\|_{*}\left\|\xi_{n}\right\|_{\mathcal{M}} \rightarrow 0
$$


Concerning $\alpha_{n}$, we have two possibilities. If $\lambda_{\star} \in\{-\infty, \infty\}$, the Riemann-Lebesgue lemma yields the convergence

$$
\alpha_{n} \rightarrow \int_{\mathbb{S}} \mu(s) \mathrm{d} s>0
$$

whereas, if $\lambda_{\star} \in \mathbb{R} \backslash\{0\}$,

$$
\mathfrak{R e} \alpha_{n} \rightarrow \int_{\mathbb{S}} \mu(s)\left(1-\cos \lambda_{\star} s\right) \mathrm{d} s>0 .
$$

In either case $\mathfrak{R e} \alpha_{n}$ has positive limit (again, UTS).

Lemma 9. The convergence $\left\|\psi_{n x}\right\| \rightarrow 0$ holds uts.

Proof. Define

$$
\zeta_{n}(s)=\frac{1}{\mathrm{i} \lambda_{n}}\left(1-\mathrm{e}^{-\mathrm{i} \lambda_{n} s}\right)\left(\tilde{\psi}_{n}-\mathrm{i} \lambda_{n} \psi_{n}\right)
$$

By means of (5.6), it is apparent that $\zeta_{n} \rightarrow 0$ in $\mathcal{M}$ and, thanks to (5.10),

$$
\eta_{n}(s)=\left(1-\mathrm{e}^{-\mathrm{i} \lambda_{n} s}\right) \psi_{n}+\int_{0}^{s} \mathrm{e}^{-\mathrm{i} \lambda_{n}(s-\sigma)} \xi_{n}(\sigma) \mathrm{d} \sigma+\zeta_{n}(s),
$$

which, on account of Lemma 5, entails

$$
\left\langle\eta_{n}, \psi_{n}\right\rangle_{\mathcal{S}}-\left\langle\zeta_{n}, \psi_{n}\right\rangle_{\mathcal{S}}=\alpha_{n}\left\|\psi_{n x}\right\|^{2}+\gamma_{n} \rightarrow 0,
$$

with $\alpha_{n}$ as above and

$$
\gamma_{n}=\int_{\mathbb{S}} \mu(s) \int_{0}^{s} \mathrm{e}^{-\mathrm{i} \lambda_{n}(s-\sigma)}\left\langle\xi_{n x}(\sigma), \psi_{n x}\right\rangle \mathrm{d} \sigma \mathrm{d} s .
$$

An application of Lemma 7 gives

$$
\left|\gamma_{n}\right| \leq \frac{\sqrt{4 m C}}{\delta}\left\|\psi_{n x}\right\|\left\|\xi_{n}\right\|_{\mathcal{M}} \rightarrow 0
$$

Knowing that $\alpha_{n}$ remains away from zero, we conclude that $\left\|\psi_{n x}\right\| \rightarrow 0$.

Lemma 10. The convergence $\left\|\eta_{n}\right\|_{\mathcal{M}} \rightarrow 0$ holds uts.

Proof. Making use of (5.11), we easily obtain the estimate $\left\|\eta_{n}\right\|_{\mathcal{M}}^{2} \leq 2 \sqrt{m}\left\|\psi_{n x}\right\|\left\|\eta_{n}\right\|_{\mathcal{M}}+\int_{0}^{\infty} \mu(s)\left\|\eta_{n x}(s)\right\| \int_{0}^{s}\left\|\xi_{n x}(\sigma)\right\| \mathrm{d} \sigma \mathrm{d} s+\left\|\zeta_{n}\right\|_{\mathcal{M}}\left\|\eta_{n}\right\|_{\mathcal{M}}$

Arguing exactly as in Lemma 7, we see that

Consequently,

$$
\int_{0}^{\infty} \mu(s)\left\|\eta_{n x}(s)\right\| \int_{0}^{s}\left\|\xi_{n x}(\sigma)\right\| \mathrm{d} \sigma \mathrm{d} s \leq \frac{\sqrt{4 C}}{\delta}\left\|\eta_{n}\right\|_{\mathcal{M}}\left\|\xi_{n}\right\|_{\mathcal{M}}
$$

$$
\left\|\eta_{n}\right\|_{\mathcal{M}} \leq 2 \sqrt{m}\left\|\psi_{n x}\right\|+\frac{\sqrt{4 C}}{\delta}\left\|\xi_{n}\right\|_{\mathcal{M}}+\left\|\zeta_{n}\right\|_{\mathcal{M}} \rightarrow 0
$$

on account of Lemma 9.

At this point, we introduce the sequence of functions

$$
F_{n}(x)=a \psi_{n x}(x)+\int_{0}^{\infty} \mu(s) \eta_{n x}(x, s) \mathrm{d} s .
$$

An asymptotic control of certain boundary terms will be needed.

Lemma 11. The convergence $F_{n}(x) \overline{\varphi_{n x}(x)} \rightarrow 0$ holds uTs for $x=0$ and $x=\ell$. 
Proof. The argument follows the lines of [7]. Accordingly, choose a real function $q \in \mathcal{C}^{1}(\mathfrak{I})$ satisfying $q(0)=-q(\ell)=1$. A multiplication of (5.7) by

$$
Q_{n}(x)=q(x) F_{n}(x)
$$

yields the convergence

$$
\mathrm{i} \lambda_{n} \rho_{2}\left\langle\tilde{\psi}_{n}, Q_{n}\right\rangle-\left\langle F_{n x}, Q_{n}\right\rangle+\kappa\left\langle\varphi_{n x}+\psi_{n}, Q_{n}\right\rangle \rightarrow 0 .
$$

By Lemmas 9 and 10, it is readily seen that $F_{n} \rightarrow 0$ in $L^{2}(\mathfrak{I})$, thus

$$
\kappa\left\langle\varphi_{n x}+\psi_{n}, Q_{n}\right\rangle \rightarrow 0 .
$$

We claim that

$$
\mathfrak{R e ~ i ~} \lambda_{n}\left\langle\tilde{\psi}_{n}, Q_{n}\right\rangle \rightarrow 0 .
$$

Indeed, exploiting (5.6) we get

$$
\mathrm{i} \lambda_{n}\left\langle q \tilde{\psi}_{n}, a \psi_{n x}\right\rangle=-a\left\langle q \tilde{\psi}_{n}, \mathrm{i} \lambda_{n} \psi_{n x}\right\rangle=-a\left\langle q \tilde{\psi}_{n}, \tilde{\psi}_{n x}\right\rangle+\varepsilon_{n}
$$

for some complex sequence $\varepsilon_{n} \rightarrow 0$, whereas (5.8) and an integration by parts yield (the boundary terms disappear as in [5])

$$
\begin{aligned}
\mathrm{i} \lambda_{n} \int_{0}^{\infty} \mu(s)\left\langle q \tilde{\psi}_{n}, \eta_{n x}(s)\right\rangle \mathrm{d} s & =-\int_{0}^{\infty} \mu(s)\left\langle q \tilde{\psi}_{n}, \mathrm{i} \lambda_{n} \eta_{n x}(s)\right\rangle \mathrm{d} s \\
& =-\int_{0}^{\infty} \mu^{\prime}(s)\left\langle q \tilde{\psi}_{n}, \eta_{n x}(s)\right\rangle \mathrm{d} s-m\left\langle q \tilde{\psi}_{n}, \tilde{\psi}_{n x}\right\rangle+\nu_{n}
\end{aligned}
$$

for some complex sequence $\nu_{n} \rightarrow 0$. Collecting the two identities, we obtain

$$
\mathrm{i} \lambda_{n}\left\langle\tilde{\psi}_{n}, Q_{n}\right\rangle=-b\left\langle q \tilde{\psi}_{n}, \tilde{\psi}_{n x}\right\rangle-\int_{0}^{\infty} \mu^{\prime}(s)\left\langle q \tilde{\psi}_{n}, \eta_{n x}(s)\right\rangle \mathrm{d} s+\varepsilon_{n}+\nu_{n} .
$$

Using Lemma 8 and integrating by parts, we infer that

$$
2 \mathfrak{R e}\left\langle q \tilde{\psi}_{n}, \tilde{\psi}_{n x}\right\rangle=\int_{0}^{\ell} q(x) \frac{\mathrm{d}}{\mathrm{d} x}\left|\tilde{\psi}_{n}(x)\right|^{2} \mathrm{~d} x=-\left\langle q^{\prime} \tilde{\psi}_{n}, \tilde{\psi}_{n}\right\rangle \rightarrow 0 .
$$

Besides, invoking (5.9),

$$
\int_{0}^{\infty} \mu^{\prime}(s)\left\langle q \tilde{\psi}_{n}, \eta_{n x}(s)\right\rangle \mathrm{d} s \rightarrow 0,
$$

and the claim is established. Summarizing, we arrive at the convergence

$$
\mathfrak{R e}\left\langle F_{n x}, Q_{n}\right\rangle \rightarrow 0 \text {. }
$$

Writing

$$
-2 \mathfrak{R e}\left\langle F_{n x}, Q_{n}\right\rangle=\left|F_{n}(\ell)\right|^{2}+\left|F_{n}(0)\right|^{2}+\left\langle q^{\prime} F_{n}, F_{n}\right\rangle,
$$

and noting that the last term in the right-hand side vanishes as $n \rightarrow \infty$, we reach the conclusion

$$
\left|F_{n}(\ell)\right|^{2}+\left|F_{n}(0)\right|^{2} \rightarrow 0
$$

To finish the proof it is now enough showing that the sequence

$$
\left|\varphi_{n x}(\ell)\right|^{2}+\left|\varphi_{n x}(0)\right|^{2}=-2 \mathfrak{R e}\left\langle q \varphi_{n x}, \varphi_{n x x}\right\rangle-\left\langle q^{\prime} \varphi_{n x}, \varphi_{n x}\right\rangle
$$

is bounded. So is certainly the second term in the right-hand side above. Concerning the first one, multiplying (5.5) by $q \varphi_{n x}$, and taking advantage of (5.4) and Lemma 9, we have

$-\rho_{1}\left\langle q^{\prime} \tilde{\varphi}_{n}, \tilde{\varphi}_{n}\right\rangle+2 \kappa \mathfrak{R e}\left\langle q \varphi_{n x}, \varphi_{n x x}\right\rangle=2 \rho_{1} \mathfrak{R e}\left\langle q \tilde{\varphi}_{n}, \tilde{\varphi}_{n x}\right\rangle+2 \kappa \mathfrak{R e}\left\langle q \varphi_{n x}, \varphi_{n x x}\right\rangle \rightarrow 0$.

Since $\left\langle q^{\prime} \tilde{\varphi}_{n}, \tilde{\varphi}_{n}\right\rangle$ is bounded, the same is true for $\mathfrak{R e}\left\langle q \varphi_{n x}, \varphi_{n x x}\right\rangle$. 
REMARK. Observe that, within the Neumann boundary condition for either $\varphi$ or $\psi$, Lemma 11 is trivially true.

Lemma 12. Within condition (1.2), the convergence $\left\|\varphi_{n x}+\psi_{n}\right\| \rightarrow 0$ holds UTS.

Proof. Multiplying (5.7) by $\varphi_{n x}+\psi_{n}$ and exploiting (5.5), we obtain

$$
\kappa\left\|\varphi_{n x}+\psi_{n}\right\|^{2}-\left.F_{n} \overline{\varphi_{n x}}\right|_{0} ^{\ell}+\mathrm{i} \lambda_{n} \rho_{2}\left\langle\tilde{\psi}_{n}, \varphi_{n x}+\psi_{n}\right\rangle-\mathrm{i} \lambda_{n} \frac{\rho_{1}}{\kappa}\left\langle F_{n}, \tilde{\varphi}_{n}\right\rangle \rightarrow 0 .
$$

The boundary term goes to zero by Lemma 11. Besides, multiplying (5.6) by $\tilde{\psi}_{n}$ and applying Lemma 8, we learn that

$$
\mathrm{i} \lambda_{n} \rho_{2}\left\langle\tilde{\psi}_{n}, \psi_{n}\right\rangle \rightarrow 0
$$

and we deduce the convergence

$$
\kappa\left\|\varphi_{n x}+\psi_{n}\right\|^{2}+\mathrm{i} \lambda_{n} \rho_{2}\left\langle\tilde{\psi}_{n}, \varphi_{n x}\right\rangle-\mathrm{i} \lambda_{n} \frac{\rho_{1}}{\kappa}\left\langle F_{n}, \tilde{\varphi}_{n}\right\rangle \rightarrow 0 .
$$

Finally, using (1.2), (5.4), (5.6) and (5.8), we have

$$
\begin{aligned}
& \mathrm{i} \lambda_{n} \rho_{2}\left\langle\tilde{\psi}_{n}, \varphi_{n x}\right\rangle-\mathrm{i} \lambda_{n} \frac{\rho_{1}}{\kappa}\left\langle F_{n}, \tilde{\varphi}_{n}\right\rangle \\
& =-\rho_{2}\left\langle\tilde{\psi}_{n}, \mathrm{i} \lambda_{n} \varphi_{n x}\right\rangle-\frac{b \rho_{1}}{\kappa}\left\langle\mathrm{i} \lambda_{n} \psi_{n x}, \tilde{\varphi}_{n}\right\rangle-\frac{\rho_{1}}{\kappa} \int_{0}^{\infty} \mu(s)\left\langle\mathrm{i} \lambda_{n} \eta_{n x}(s)-\mathrm{i} \lambda_{n} \psi_{n x}, \tilde{\varphi}_{n}\right\rangle \mathrm{d} s \\
& =b\left(\frac{\rho_{1}}{\kappa}-\frac{\rho_{2}}{b}\right)\left\langle\tilde{\psi}_{n}, \tilde{\varphi}_{n x}\right\rangle-\frac{\rho_{1}}{\kappa} \int_{0}^{\infty} \mu^{\prime}(s)\left\langle\eta_{n x}(s), \tilde{\varphi}_{n}\right\rangle \mathrm{d} s+\varepsilon_{n} \\
& =-\frac{\rho_{1}}{\kappa} \int_{0}^{\infty} \mu^{\prime}(s)\left\langle\eta_{n x}(s), \tilde{\varphi}_{n}\right\rangle \mathrm{d} s+\varepsilon_{n},
\end{aligned}
$$

for some complex sequence $\varepsilon_{n} \rightarrow 0$. Since (5.9) bears

$$
\int_{0}^{\infty} \mu^{\prime}(s)\left\langle\eta_{n x}(s), \tilde{\varphi}_{n}\right\rangle \mathrm{d} s \rightarrow 0
$$

the proof is finished.

On account of Lemmas 8, 9, 10 and 12, the sought contradiction is attained once we prove the convergence $\left\|\tilde{\varphi}_{n}\right\| \rightarrow 0$ UTS. To this end, a multiplication of (5.5) by $\varphi_{n}$ will do.

\section{References}

[1] F. Ammar Khodja, A. Benabdallah, J.E. Muoz Rivera and R. Racke, Energy decay for Timoshenko systems of memory type, J. Differential Equations 194 (2003), 82-115.

[2] V.V. Chepyzhov and V. Pata, Some remarks on stability of semigroups arising from linear viscoelasticity, Asymptot. Anal. 46 (2006), 251-273.

[3] C.M. Dafermos, Asymptotic stability in viscoelasticity, Arch. Rational Mech. Anal. 37 (1970), 297-308.

[4] C. Giorgi, M.G. Naso and V. Pata, Exponential stability in linear heat conduction with memory: a semigroup approach, Comm. Appl. Anal. 5 (2001), 121-134.

[5] M. Grasselli and V. Pata, Uniform attractors of nonautonomous systems with memory, in "Evolution Equations, Semigroups and Functional Analysis" (A. Lorenzi and B. Ruf, Eds.), pp.155-178, Progr. Nonlinear Differential Equations Appl. no.50, Birkhäuser, Boston, 2002.

[6] S.A. Messaoudi and B. Said-Houari, Uniform decay in a Timoshenko-type system with past memory, J. Math. Anal. Appl. 360 (2009), 459-475.

[7] J.E. Muñoz Rivera and H.D. Fernández Sare, Stability of Timoshenko systems with past history, J. Math. Anal. Appl. 339 (2008), 482-502. 
[8] J.E. Muñoz Rivera and R. Racke, Mildly dissipative nonlinear Timoshenko systems-global existence and exponential stability, J. Math. Anal. Appl. 276 (2002), 248-278.

[9] V. Pata, Exponential stability in linear viscoelasticity with almost flat memory kernels, Commun. Pure Appl. Anal. 9 (2010), 721-730.

[10] J. Prüss, On the spectrum of $C_{0}$-semigroups, Trans. Amer. Math. Soc. 284 (1984), 847-857.

[11] A. Soufyane, Stabilisation de la poutre de Timoshenko, C. R. Acad. Sci. Paris Sér. I Math. 228 (1999), 731-734.

[12] S.P. Timoshenko, On the correction for shear of a differential equation for transverse vibrations of prismatic bars, Philos. Magazine 41 (1921), 744-746.

Politecnico di Milano - Dipartimento di Matematica "F. Brioschi" Via Bonardi 9, 20133 Milano, Italy

E-mail address: monica.conti@polimi.it

Politecnico di Milano - Dipartimento di Matematica "F. Brioschi" Via Bonardi 9, 20133 Milano, Italy

E-mail address: filippo.delloro@mail.polimi.it

Politecnico di Milano - Dipartimento di Matematica "F. Brioschi" Via Bonardi 9, 20133 Milano, Italy

E-mail address: vittorino.pata@polimi.it 